\title{
Capitalisme et catholicisme dans la France moderne. La dynastie Le Couteulx
}

Guy Lemarchand

\section{Q OpenEdition \\ 1 Journals}

Édition électronique

URL : https://journals.openedition.org/ahrf/2255

DOI : 10.4000/ahrf.2255

ISSN : 1952-403X

\section{Éditeur :}

Armand Colin, Société des études robespierristes

\section{Édition imprimée}

Date de publication : 1 juin 2002

Pagination : 225-228

ISSN : 0003-4436

\section{Référence électronique}

Guy Lemarchand, «Capitalisme et catholicisme dans la France moderne. La dynastie Le Couteulx », Annales historiques de la Révolution française [En ligne], 328 | avril-juin 2002, mis en ligne le 11 mai 2006, consulté le 23 avril 2022. URL : http://journals.openedition.org/ahrf/2255 ; DOI : https://doi.org/ 10.4000/ahrf.2255

Ce document a été généré automatiquement le 23 avril 2022.

Tous droits réservés 


\title{
Capitalisme et catholicisme dans la France moderne. La dynastie Le Couteulx
}

\author{
Guy Lemarchand
}

\section{RÉFÉRENCE}

Michel Zylberberg, Capitalisme et catholicisme dans la France moderne. La dynastie Le

Couteulx, Paris, publications de la Sorbonne, 2001, 380 p.

1 S'agit-il avec ce livre d'une monographie de plus de famille bourgeoise à l'époque moderne? Tout en s'inscrivant dans la lignée des recherches consacrées aux dynasties de grands marchands et entrepreneurs industriels à la suite des Danse et Motte de Beauvais de P. Gombert (1959) et des Dolle et Raby de Grenoble de P.Léon (1963), M.Zylberberg a voulu aller plus loin à propos de ces négociants occupés surtout de banque, d'origine normande, installés tôt à Paris et qui vont être parmi les maîtres de la finance privée. D'emblée l'auteur pose les grandes questions de l'historiographie récente: hors des grands ports comme Saint-Malo sous Louis XIV étudié par A. Lespagnol (1990) ou Marseille au XvIII ${ }^{e}$ siècle selon C. Carrière (1973), y a-t-il eu en France une bourgeoisie d'affaires catholique active à côté des protestants connus par les travaux d'H.Luthy (1959-1961) ? Peut-on distinguer une culture intellectuelle et un comportement social dans l'Ancien Régime propres à cette bourgeoisie? La Révolution s'est-elle faite sans elle comme le voulaient A. Cobban (1964), G. Taylor (1967) et divers historiens français à leur suite? On débouche ainsi sur une importante controverse réveillée, il y a douze ans, par le bicentenaire de 1789. Et pourtant M. Zylberberg disposait à la fois de très peu et de trop de sources. Très peu : aucun des grands livres de comptabilité, tous disparus, pas de liasses d'effets de commerce ni de facturiers, pas de livre de raison ni de correspondance abondante, seulement des généalogies d'époque et quelques contrats de mariage et actes de succession. Trop de documents : en face de ces lacunes, outre les Archives départementales de Seine-Maritime, le 
Minutier central des Archives nationales et les papiers des gouvernements et assemblées révolutionnaires, l'auteur est allé à la quête de toute source partout hors de Rouen et Paris où les Le Couteulx pouvaient avoir eu des relations épistolaires et avoir passé transactions : la Bretagne, Marseille, Bayonne, Madrid, Séville, Cadix, sans oublier les documents imprimés, du traité d'économie de Montchrestien (1615) aux papiers de Th. Jefferson. Un travail considérable donc.

2 Les Le Couteulx viennent d'une famille de laboureur du sud du Pays de Caux. À la fin du $\mathrm{XV}^{\mathrm{e}}$ siècle l'un d'eux s'établit marchand à Yvetôt, en rapport par là avec Harfleur et le commerce de mer, comme souvent à l'origine des grandes fortunes. Vers 1520 un fils va vivre à Rouen, puis en 1570 Guillaume I, marchand modeste de la ville, est néanmoins déjà, par le négoce du drap, en relation avec la foire du Lendit où il tient loge, et avec l'Angleterre. En 1613, Jaques I se marie avec la fille d'un marchand drapier de Paris et il fonde l'établissement Le Couteulx et Compagnie de la capitale, rue Saint-Denis. Malgré des difficultés après 1690, dues aux crises économiques et démographiques et à l'instabilité monétaire, la famille résiste à la dépression sous Louis XIV, sans doute parce qu'elle sait diversifier son activité qualitativement et géographiquement et qu'à partir de 1670, sans abandonner la marchandise, elle s'oriente vers la banque. À la fin du XVII ${ }^{\mathrm{e}}$ siècle l'entreprise est une société familiale en nom collectif à deux établissements, Paris et Rouen, disposant de fonds propres élevés (900000 1.). Elle pratique le service de caisse de particuliers riches (perception et transfert de rentes, de gages d'officiers...), elle couvre par son crédit les importations de denrées et surtout de laine d'Espagne pour l'industrie textile de Normandie et du Nord, elle prête aux manufacturiers pour garnir leurs fonds de roulement destinés au paiement des artisans, elle achète des espèces métalliques ibériques pour les hôtels des monnaies, elle participe aux sociétés d'assurances maritimes et entretient un trafic de lettres de change d'envergure internationale qui est probablement devenu son activité principale. Au XVIII ${ }^{\mathrm{e}}$ siècle, à la tête d'un capital de 1,2 à 1,5 m. 1., elle étend encore ses affaires et crée deux sociétés filiales, l'une à Cadix (1724), l'autre à Amsterdam (1762), places qui sont les poumons de la richesse de nombreux marchands français. Elle travaille essentiellement à la commission à taux bas de moins de $1 \%$, mais le profit demeure probablement élevé (jusqu'à $25 \%$ pour la moisson de Cadix en 1738) par la masse des affaires traitées : placement des rentes viagères perpétuelles sur l'Hôtel de Ville, des rentes sur les aides, prêts à la grosse, constitution de sociétés d'armement maritime... La filiale espagnole, qui est une des plus grosses entreprises étrangères de Cadix, devient essentielle, ajoutant aux activités bancaires l'import-export, cochenille d'Amérique latine contre textiles français, sans parler, toujours, du trafic des piastres.

3 À partir du ministère Necker, les Le Couteulx se lancent dans la finance publique, d'autant que la guerre d'Indépendance américaine oblige les gouvernements à chercher de nouvelles ressources. Ils concourent au placement des emprunts de Louis XVI et à partir de 1781 des «vales reales» de Charles III. En même temps ils entrent au Conseil d'administration de la Caisse d'escompte lors de sa création en 1776 et deviennent actionnaires de la banque Saint-Charles de Madrid, ce qui permet à la maison de Cadix d'accroître ses opérations sur les piastres. Ils prêtent aussi au Congrès continental des États-Unis et leurs relations avec le superintendant Robert Morris sont l'occasion de développer les importations du tabac de Virginie. Dans la dernière décennie de l'Ancien Régime également, ils prennent le contrôle de grandes entreprises manufacturières 
telles que la fonderie de cuivre de Romilly-sur-Andelle en Normandie et la manufacture de tabac de Morlaix.

4 L'enrichissement de la famille a été presque continu du $\mathrm{XVI}^{\mathrm{e}}$ au XVIII ${ }^{\mathrm{e}}$ siècle. Ainsi la fortune des dirigeants de la société-mère passe-t-elle de 100000 l. en 1651 pour Jean IV à 648000 l. en 1721 pour Bartélémy II, à plus d'un million en 1750 pour Jean X et le parcours social du groupe, alliances, patrimoine, train de vie, est des plus classiques, marqué par un souci de sécurité et de considération mais sans ambition insatiable. La stratégie familiale évolue et aide à la croissance de la fortune : au XVII ${ }^{\mathrm{e}}$ siècle les garçons épousent des filles de commerçants, principalement des marchands-drapiers; au XVIII ${ }^{\mathrm{e}}$ siècle ils se marient dans la famille en dépit de la consanguinité, ce qui contribue à la stabilité de la richesse. De plus, alors qu'auparavant on réinvestissait les profits dans l'entreprise, à partir du début du xvIII ${ }^{e}$ siècle on multiplie les achats d'hôtels urbains et de terres et, après 1750, de châteaux et seigneuries, tandis que les dépenses somptuaires, argenterie, pendules, carrosses, rares avant 1660, vont bon train, particulièrement après 1720. Apparus sous LouisXIV, tapisseries, tableaux, livres deviennent nombreux, et au siècle des Lumières, s'ils ne sont membres d'aucune académie, pas plus que la plupart des autres négociants français, les Le Couteulx lisent des ouvrages scientifiques et la littérature philosophique, adhèrent à la franc-maçonnerie et organisent à la fin du siècle des réceptions mondaines dans leurs «folies» près de Paris. En matière religieuse, comme de nombreux marchands, notamment ceux qui trafiquent avec la mer du Nord, la famille a été tentée par le calvinisme dans les années 1560 , mais elle en sort probablement au début du XVII siècle, adhérant moralement ensuite à la Contre-Réforme et donnant même à l'Église quelques-uns de ses fils et filles. Toutefois, au XVIII ${ }^{e}$ siècle, d'après les testaments et les postes de marguilliers occupés, ce zèle se refroidit et il n'y a plus de goût pour la carrière ecclésiastique. La déchristianisation décidément n'est pas qu'une invention du complot de Michel Vovelle et de quelques mauvais sujets de cette sorte! Sur le plan des valeurs sociales les Le Couteulx ne sont pas trop pressés de «trahir la bourgeoisie». À côté des associés de la banque, les autres membres de la famille demeurent fidèles à la condition confortable de marchands-drapiers, peu achètent des offices donnant accès au second ordre et il faut attendre 1702 pour en voir un acquérant une charge de secrétaire du roi et même 1756 pour voir deux des banquiers anoblis par lettres, ce qui leur donne, semble-t-il, davantage de plaisir qu'ils n'affectent de le dire, mais, pour autant, on n'observe pas chez eux de mariages avec des filles nobles et si les trois dirigeants de la maison de Rouen participent en 1789 à l'assemblée de la noblesse du bailliage, c'est par le Tiers que Jean-Bartélémy se fait élire député aux États Généraux, tandis que Bartélémy-Jean-Louis devient membre du Comité permanent de Paris par, encore, le suffrage du Tiers de la ville. En ce qui concerne l'attitude politique les banquiers passent du loyalisme parfait au XVII e siècle à d'abord, une prise de distance critique sans remise en cause de l'ordre régnant, l'enrichissement de l'entreprise donnant à la fois aplomb et occasion de heurts avec la politique du gouvernement : à propos des billets de monnaie émis par le roi à partir de 1701 qui perturbent le change, ou du gigantisme dévorant de la Compagnie des Indes de Law. Au milieu du XVIII ${ }^{\mathrm{e}}$ siècle les Le Couteulx soutiennent le courant économique libéral de Gournay mais, on le sait, des ministres l'appuient également, et les banquiers ont même l'oreille de L'Averdy. La rupture avec la monarchie et l'exigence de réformes étendues ne surviennent qu'avec le traité de commerce avec l'Angleterre de 1786 adopté sans consultation du négoce et qui ruine l'industrie normande. Par conséquent déjà deux grandes conclusions 
s'imposent. Il y a bien une grande bourgeoisie d'affaires catholique, opulente et, pour reprendre le débat suscité par $\mathrm{M}$. Weber, d'esprit capitaliste. La fortune des Le Couteulx et Cie s'est bâtie sur les agios, les remises et les commissions ainsi que, en partie, le trafic avec les Provinces-Unies et la Grande-Bretagne, protestantes toutes deux, sans oublier d'ailleurs Genève. Second enseignement : sur le plan culturel cette bourgeoisie passe du conformisme strict, assez austère et étriqué, au XvII ${ }^{e}$ siècle (Daniel I en 1625 n'a qu'un livre et il s'agit d'une Bible, JacquesI en 1652 en a 200 mais essentiellement religieux), à une véritable autonomie et une ouverture nouvelle au XvIII ${ }^{\mathrm{e}}$ siècle que marque la bibliothèque d'Antoine-Louis avec 3540 ouvrages en 1779.

5 Pendant la Révolution et l'Empire les Le Couteulx accèdent à la scène publique nationale, alors que dans l'Ancien Régime ils s'étaient contentés de fonctions locales, juge consulaire de Rouen pour la première fois en 1619, échevin de la ville en 1644, député du Bureau de commerce de Paris en 1752, maire de Rouen en 1764, premier président de la Chambre des comptes de Normandie en 1767. Ils adhèrent fortement à 1789. À la Constituante Jean-Bartélémy, proche de Sieyès, est favorable à la nationalisation des biens du clergé, tout en cherchant à limiter les émissions d'assignats et il adhère aux Feuillants en juillet 91 ; Jacques-Jean est trésorier de la Caisse de l'Extraordinaire en 1790-1792 et Bartélémy-Jean-Louis conseiller général de l'Oise en 1792. Après avoir prêté des fonds pour la tentative de sauvetage de Louis XVI menée par l'ambassadeur d'Espagne en octobre 1792, les Le Couteulx de Paris sont arrêtés en novembre 1793 mais la branche rouennaise est épargnée. L'inflation et la guerre atteignent durement la banque mais, libéré au lendemain de Thermidor, Jacques-Jean se lance dans de fructueuses spéculations immobilières dans Paris et figure en 1806 parmi les trente plus imposés de la Seine, tandis que Jean-Bartélémy transforme la société Le Couteulx et Cie en société de portefeuille qui achète des actions de diverses compagnies. Député des Anciens en 1796, président du département de la Seine en 1799, ce dernier qui connait Bonaparte depuis 1796, soutient le coup de Brumaire et est l'un des banquiers à l'origine de la fondation de la Banque de France dont il est régent dès 1801. Après avoir maintenu de bonnes relations avec Napoléon, et avoir été fait comte en 1808, il n'en est pas moins érigé pair de France par Louis XVIII. Par ailleurs la fortune des uns et des autres a été un moment consolidée par de vastes achats de biens nationaux. Par conséquent une troisième conclusion de l'ouvrage apparaît fortement: la bourgeoisie d'affaires s'est mêlée de la politique nationale à partir de 1789 , soutenant les grandes réformes et elle a joué un rôle important jusqu'à la Restauration.

6 Un caractère particulier marque cette dynastie: sa grande prudence. Bien que la pauvreté des sources ne permette pas de se prononcer clairement, les Le Couteulx innovent peu sur le plan technique: la comptabilité en partie double n'est adoptée qu'en 1785. Ils ne sont pas plus hardis dans la recherche de nouveaux gisements de profits: ils ne participent pas aux grandes aventures commerciales, demeurant en dehors du commerce des mers du Sud au début du XviII ${ }^{e}$ siècle, de la tentative bancaire et commerciale de Law, de la première Compagnie des Indes orientales. Leurs interventions dans les finances royales sont tardives et limitées et leur intérêt pour la grande industrie ne se manifeste qu'à la veille de 1789 et ils ne possèdent pas de plantations aux Antilles. Leur domaine par excellence est le change et le crédit à court terme, l'argent qui fait vite de l'argent. Il s'agit d'une bourgeoisie sage qui attend que les nouveautés aient montré leur rentabilité et leur durabilité pour s'y lancer, ce qui 
d'ailleurs permet sa longévité en lui évitant les grands revers et les faillites. J'ajoute qu'on peut se demander s'il ne s'agit pas d'une particularité française liée à une forte densité de population qui assure des débouchés faciles, et à un climat moral où pèsent malgré tout le modèle nobiliaire et le catholicisme peu favorables aux ascensions marchandes spectaculaires ? En tous cas l'un des mérites du livre de M.Zylberberg est de replacer la famille étudiée dans son contexte qu'il décrit toujours avec précision et largement, et de lier ensemble histoire sociale et histoire économique qu'on tend assez souvent aujourd'hui à séparer soigneusement conformément à l'empirisme ambiant : ainsi l'expansion de l'industrie de la laine dans la France du Nord de la première moitié du XvI ${ }^{e}$ siècle, la croissance de la place économique de Paris au XvII ${ }^{e}$ siècle, le rôle de Cadix plaque tournante au XVIII siecle de la redistribution des produits et du métal précieux de l'Amérique espagnole, le poids direct de la politique extérieure et intérieure sur les affaires à partir de 1792. Depuis une douzaine d'années, sous l'influence entre autres de P.Ricœur, certains historiens mettent en avant l'acteur et la mise en intrigue, tendant par là à privilégier l'individu dans son caractère unique et le pouvoir de l'action à court terme en elle-même et opposant de telles découvertes à l'histoire sociale classique déclarée ringarde et répétitive. M. Zylberberg ici, en dépit des importantes lacunes dans les sources, en se plaçant sous l'angle du collectif et de la longue durée, dresse tout de même le portrait de quelques figures vivantes et dessine l'ample comédie au sens balzacien du terme qu'est la montée et la puissance de la famille Le Couteulx. Chemin faisant, il traite de grands problèmes en utilisant les bonnes vieilles méthodes de l'histoire quantitative, mais élargies et affinées depuis quarante ans. Donc une réussite. 